\title{
Chain Length Effects of Linear Alkanes in Zeolite Ferrierite. 2. Molecular Simulations ${ }^{1}$
}

\author{
Willy J. M. van Well, ${ }^{\dagger}$ Xavier Cottin, ${ }^{\dagger}$ Berend Smit, ${ }^{\ddagger}$ Jan H. C. van Hooff, ${ }^{\dagger}$ and \\ Rutger A. van Santen*, \\ Schuit Institute of Catalysis, Laboratory for Inorganic Chemistry and Catalysis, Eindhoven University of \\ Technology, P.O. Box 513, 5600 MB Eindhoven, The Netherlands; and Department of Chemical Engineering, \\ University of Amsterdam, Nieuwe Achtergracht 166, 1018 WV Amsterdam, The Netherlands
}

Received: December 3, 1997; In Final Form: March 5, 1998

\begin{abstract}
Configurational-bias Monte Carlo technique simulations are performed in the grand canonical ensemble to calculate the adsorption isotherms and the isosteric heats of adsorption of propane, $n$-butane, $n$-pentane, and $n$-hexane in ferrierite. The results are generally in good agreement with experiment results. The locations of the molecules in the ferrierite pore structure obtained from the simulations agree well with the locations obtained by earlier ${ }^{13} \mathrm{C}$ NMR measurements. It is found that only short $n$-alkane molecules up to $n$-pentane can access the entire two-dimensional ferrierite pore structure; longer molecules adsorb only in the 10-ring channels and not in the ferrierite 8-ring cage. Adsorption of $n$-pentane in this cage is possible but only at relatively high pressures due to the high number of repulsive interactions with framework oxygen ions. The results presented are furthermore used to set limits to the size parameter describing the Lennard-Jones interaction between the $n$-alkane united atoms and the framework oxygen atoms.
\end{abstract}

\section{Introduction}

Knowledge of the adsorption properties of reactants, intermediates, and products is a prerequisite for a thorough understanding and optimization of the catalytic performance of zeolite catalysts. The use of traditional experimental techniques is often not sufficient to obtain a full knowledge and understanding of these properties. It is furthermore especially difficult to study the adsorption of large and of long chain adsorbates and to perform adsorption studies at reaction conditions. Moreover, some important properties such as location and conformation of the adsorbed molecules are, even for small molecules and moderate conditions, hard to obtain experimentally. For these reasons, significant effort has been devoted to the development of molecular simulation techniques that are able to predict adsorption properties within reasonable computation time. The configurational-bias Monte Carlo (CB-MC) technique is a result of these efforts and has become a valuable tool in the research field of adsorption in zeolites. In contrast to the usual Monte Carlo methods, where an adsorbate molecule is placed at once into the zeolite, the molecules are grown atom by atom in the $\mathrm{CB}-\mathrm{MC}$ technique. This makes the $\mathrm{CB}-\mathrm{MC}$ technique orders of magnitude more efficient than traditional Monte Carlo methods for the simulation of the adsorption of long chain molecules.

The CB-MC technique was used by Smit et al. to study the adsorption of linear alkanes from methane to dodecane into allsilica ZSM-5, silicalite. $^{2-5}$ Both adsorption properties at infinite dilution and adsorption isotherms were calculated. Besides energetic properties, the location and conformation of the alkane molecules were also obtained from the simulations. Maginn et al. were able to predict the adsorption properties in silicalite of linear alkanes as long as pentacosane at infinite dilution at temperatures as high as $800 \mathrm{~K}^{6}{ }^{6}$ The energetics, location, and

\footnotetext{
Eindhoven University of Technology.

$\doteqdot$ University of Amsterdam.
}

conformation of linear alkanes from butane to decane adsorbed at infinite dilution in a range of zeolites with different pore structures were studied recently by Bates and co-workers. ${ }^{7,8}$ Finally, the effect of temperature and $n$-butane concentration on the conformation of $n$-butane adsorbed in zeolites $\mathrm{NaY}$ and $\mathrm{NaCaA}$ was studied by means of CB-MC by Bandyopadhyay and Yashonath. ${ }^{9,10}$ The examples mentioned above show the usefulness of molecular simulations. Besides the ability to calculate properties that are not easily accessible by experimental methods, they are also able to obtain a better understanding of the experimental results. The adsorption of $n$-alkanes in silicalite is an excellent example where simulations have given a detailed molecular interpretation of results from experiments. In the adsorption isotherms measured experimentally, a step in the dependence of pressure was found exclusively for $n$-hexane and $n$-heptane, whereas for longer and shorter $n$-alkanes the normal type I isotherm was measured. This step occurred also exclusively for $n$-hexane and $n$-heptane in the calculated isotherms in silicalite of Smit et al. ${ }^{5}$ But more importantly, the authors were also able to give an explanation of this phenomenon by analyzing the location of the molecules in the silicalite pores. They found that, at about half the maximum pore filling, all $n$-hexane and $n$-heptane molecules move into the zigzag channels of silicalite. Since the $n$-hexane and $n$-heptane molecules have approximately the same length as one segment of the zigzag channels, the straight channels become completely free for further adsorption. The movement of the molecules into the zigzag channels results finally in an optimum filling of the pores by $n$-hexane and $n$-heptane. An interesting fundamental question is whether this phenomenon is unique for silicalite, or whether similar effects can be expected within other zeolites.

We will study here the adsorption properties of linear alkanes in the zeolite ferrierite. The ferrierite-catalyzed $n$-butene isomerization is an important process of which several aspects are still unclear. ${ }^{11}$ Knowledge and understanding of the 
adsorption properties of hydrocarbons in ferrierite could serve to elucidate some of these aspects. Investigations of the adsorption properties in ferrierite are furthermore interesting because its two-dimensional pore structure might cause similar phenomena as found in the case of silicalite. In part 1, we have studied experimentally the adsorption properties and location of linear alkanes in ferrierite. ${ }^{12}$ We will compare the results obtained from CB-MC simulations to these experimental results and use them to get a better understanding of the origins of the remarkable adsorption behavior of linear alkanes in ferrierite. Furthermore, the parameters used in the CB-MC simulations to describe the interaction between the zeolite framework and the alkane molecules will be tested by the comparison of results obtained from simulations and experiments. These parameters have been derived form adsorption data of linear alkanes in silicalite. $^{4,5,13}$ It is therefore important to have a solid test of these parameters on other zeolites.

\section{Simulation Details}

The CB-MC method as developed by Smit and Siepmann is used in this work; a detailed description of this technique can be found elsewhere. ${ }^{3}$ To enable the calculation of adsorption isotherms, the simulations are performed in the grand-canonical ensemble. This means that the chemical potential, temperature, and volume are fixed during the simulation. The use of CBMC simulations in the grand-canonical ensemble to calculate adsorption isotherms is discussed in refs 14 and 15 .

The simulations of the adsorption isotherms were performed in equilibration and production cycles. At least $5 \times 10^{4}$ equilibration cycles and at least $5 \times 10^{4}$ production cycles were used. Each cycle consists of attempts to perform different Monte Carlo moves with a certain probability. For propane, the probabilities for the different moves were such that $25 \%$ of the attempts were a displacement of a molecule, $25 \%$ a rotation, $10 \%$ partly regrow of a molecule, and $40 \%$ addition or removal of a molecule. The number of trial orientations for the last two moves was 4 . The probability to displace or to rotate a molecule was $25 \%$ for $n$-butane and longer molecules, and the probability for a partly regrow attempt was $20 \%$, leaving $30 \%$ of the attempts for addition or removal of a molecule. Seven trial orientations were used for the last two moves. The probability to add or remove a molecule was increased when the number of successful molecule swaps between the reservoir and the system became low. An increase of this probability was especially necessary for higher loadings of the longer molecules.

The $n$-alkanes are modeled using the united atom model; this means that $\mathrm{CH}_{3}$ and $\mathrm{CH}_{2}$ units are treated as single interaction centers. The bond length between these pseudoatoms has been kept fixed. The bond bending is described by a harmonic potential while the torsional angles are modeled by the Jorgensen potential, and the interactions between different pseudoatoms are described by a $12-6$ Lennard-Jones potential. This model has been used to describe successfully the vapor-liquid phase equilibria of $n$-alkanes over a large temperature range. ${ }^{16}$ Further details of the $n$-alkane models are given for propane in ref 4 and for $n$-butane and longer $n$-alkanes in ref 3 .

The ferrierite structure as determined by Vaughan ${ }^{17}$ has been used and kept rigid in the simulations. All aluminum atoms were replaced by silicon atoms since only the dispersive interactions between the hydrocarbon and the zeolite framework are taken into account. We have used a simulation box that consists of 36 unit cells ( 2 in the $a$-direction, 3 in the $b$-direction and 6 in the $c$-direction). The dimensions of the simulation box are $a=38.312 \AA, b=42.381 \AA$, and $c=44.934 \AA$.
TABLE 1: Size and Energy Parameters of the Different Parameter Sets Used Describing the Interaction between the Framework Oxygen Atoms and the Alkane United Atoms

\begin{tabular}{lccc}
\hline & $\sigma_{\mathrm{CH} 3, \mathrm{O}}=\sigma_{\mathrm{CH} 2, \mathrm{O}}[\AA]$ & $\epsilon_{\mathrm{CH} 3, \mathrm{O}}[\mathrm{K}]$ & $\epsilon_{\mathrm{CH} 2, \mathrm{O}}[\mathrm{K}]$ \\
\hline propane & 3.636 & 73.4 & 73.4 \\
Smit et al. & 3.64 & 87.5 & 54.4 \\
June et al. & 3.364 & 83.8 & 83.8
\end{tabular}

Periodic boundary conditions have been imposed during simulation. A more detailed description of the ferrierite structure is given in part $1 .{ }^{12}$

Only the interaction between the oxygen atoms of the framework and the united atoms describing the alkane chains has been considered. These dispersive interactions are described by a $12-6$ Lennard-Jones potential:

$$
U_{\mathrm{LJ}}=4 \epsilon_{i j}\left[\left(\sigma_{i j} / r_{i j}\right)^{12}-\left(\sigma_{i j} / r_{i j}\right)^{6}\right]
$$

The size $(\sigma)$ and energy $(\epsilon)$ parameters of this potential for $n$-butane and longer molecules are derived from experimentally determined heats of adsorption and Henry coefficients of $n$-alkanes, ranging from $n$-butane to $n$-decane, in silicalite. ${ }^{5}$ For propane, these parameters are derived from experimental adsorption data of propane in silicalite. ${ }^{4}$ These two sets of parameters are given in Table 1 and denoted as the parameter set of Smit et al. and as the Propane parameter set.

Another set of parameters for this Lennard-Jones potential describing the interaction between the zeolite framework and $n$-butane and longer $n$-alkanes has been derived by June et al. ${ }^{13}$ These parameters are also given in Table 1 . We have used these parameters in some of the calculations to enable a comparison with the parameters of Smit et al. and to enable a test of the different sets of parameters. The zeolite-alkane potential in all three models is truncated at $13.8 \AA$, and the usual tail corrections have been applied.

Besides the adsorption isotherms, the isosteric heat of adsorption $q_{\text {st }}$ has also been calculated. In the limit of zero coverage, this property is simply the difference between the enthalpy of an adsorbed alkane molecule and the enthalpy of an alkane molecule in the ideal gas state. ${ }^{18}$ The latter quantity can be calculated by means of a separate simulation without zeolite lattice. ${ }^{3}$ To calculate the isosteric heat of adsorption at loadings above infinite dilution, we have used the formula derived by Vuong and Monson: ${ }^{19}$

$$
q_{\mathrm{st}}=H^{(\mathrm{b})}-\left(\frac{\partial U^{(\mathrm{a})}}{\partial N^{(\mathrm{a})}}\right)_{T, V^{(\mathrm{a})}}
$$

In this formula, $U^{(\text {a) }}$ stands for the total energy of the adsorbed molecules and $N^{(a)}$ for the total number of adsorbed molecules. The partial derivative of the total energy is calculated using fluctuation theory as

$$
\left(\frac{\partial U}{\partial N}\right)_{T, V}=\frac{\langle U N\rangle-\langle U\rangle\langle N\rangle}{\left\langle N^{2}\right\rangle-\langle N\rangle^{2}}
$$

The brackets in this equation denote averages over configurations. Thus $\left\langle N^{2}\right\rangle$ stands for the average of the number of adsorbed molecules squared, while $\langle N\rangle^{2}$ is the square of the average number of adsorbed molecules. The enthalpy of the bulk phase $\mathrm{H}^{(\mathrm{b})}$ is calculated with the equation of state of Lee and Kesler. ${ }^{20}$ This equation of state gives the difference in enthalpy of a molecule in the bulk phase and a molecule in the 


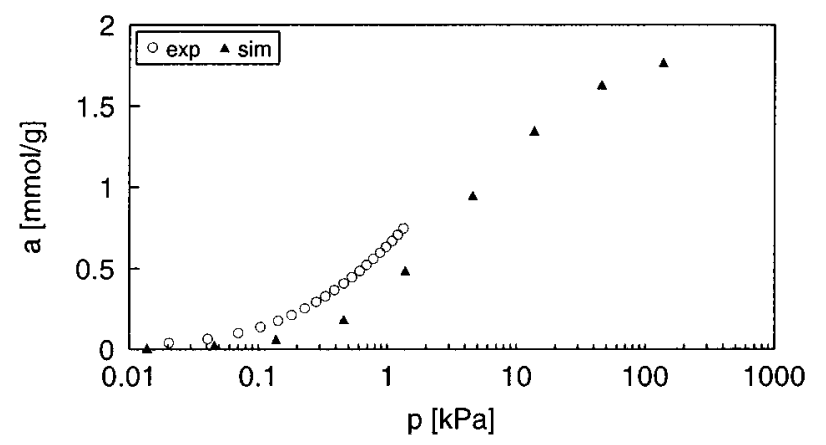

Figure 1. Simulated and experimental adsorption isotherm of propane in FER at $333 \mathrm{~K}$. The simulated isotherm is determined on $\mathrm{SiO}_{2}$ FER using the Propane parameter set; the experimental isotherm is determined on H-FER. ${ }^{21}$

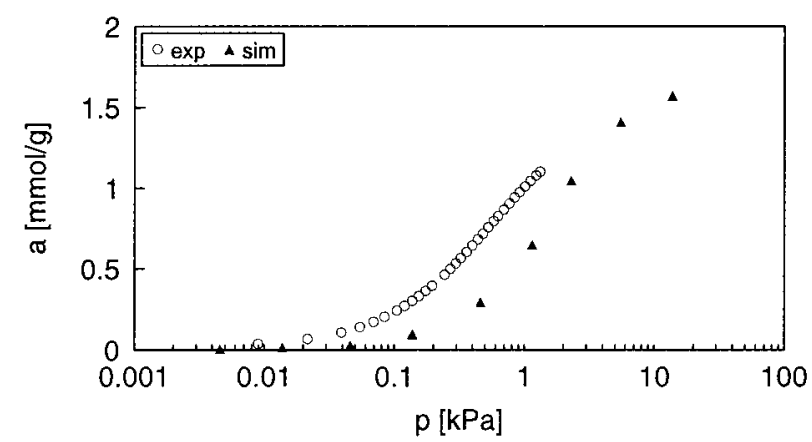

Figure 2. Simulated and experimental adsorption isotherm of $n$-butane in FER at $333 \mathrm{~K}$. The simulated isotherm is determined on $\mathrm{SiO}_{2}$ FER using the Smit et al. parameter set; the experimental isotherm is determined on H-FER. ${ }^{21}$

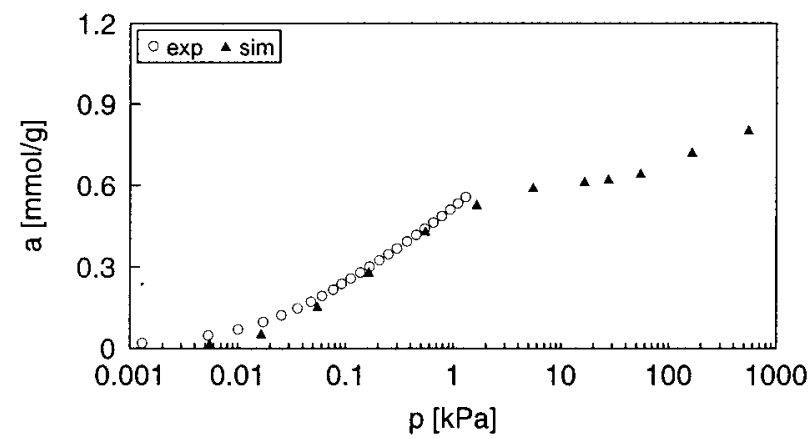

Figure 3. Simulated and experimental adsorption isotherm of $n$-pentane in FER at $333 \mathrm{~K}$. For more details see caption to Figure 2.

ideal gas state. By calculation of the latter property through simulation (see above), the enthalpy of the bulk phase is obtained.

\section{Simulation Results and Discussion}

Adsorption Isotherms at $\mathbf{3 3 3} \mathbf{K}$. The adsorption isotherms of propane, $n$-butane, $n$-pentane, and $n$-hexane in ferrierite have been calculated using the Smit et al. and the Propane parameter set at $333 \mathrm{~K}$. The results are given in Figures 1-4 and compared to the experimental isotherms of Eder et al. ${ }^{21}$ An almost perfect agreement between the simulation results and the experimental results can be seen for $n$-pentane and $n$-hexane in Figures 3 and Figure 4. The agreement is less for propane and $n$-butane; the loadings calculated are, at a given pressure, lower than the loadings measured experimentally.

Both simulations and experiments show that propane and $n$-butane can reach high loadings at relatively low pressures, while $n$-hexane fills the ferrierite framework only partly. High

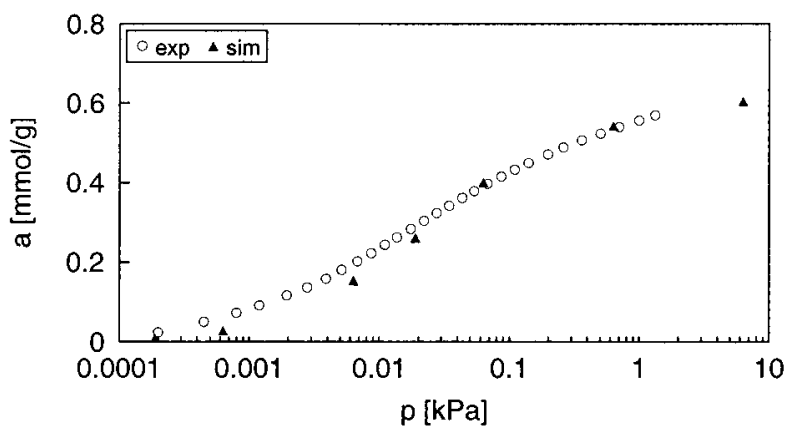

Figure 4. Simulated and experimental adsorption isotherm of $n$-hexane in FER at $333 \mathrm{~K}$. For more details see caption to Figure 2.

loadings of $n$-hexane are not even found when the simulations of the $n$-hexane adsorption isotherm are extended up to a pressure of $6300 \mathrm{kPa}$. The simulated isotherm of $n$-pentane has a huge step at a loading of about $0.6 \mathrm{mmol} / \mathrm{g}$ from $5.5 \mathrm{kPa}$ to $55 \mathrm{kPa}$. Complete pore filling with $n$-pentane is, according to the simulations, not possible at pressures below the pressure at the critical point. The $n$-pentane loading amounts to 0.81 $\mathrm{mmol} / \mathrm{g}$ at a pressure of $550 \mathrm{kPa}$, which is just below the critical point. Higher loadings can be reached when the simulations are continued at higher pressures; for example, at $5500 \mathrm{kPa}$ the loading calculated is $1.4 \mathrm{mmol} / \mathrm{g}$.

In part 1, we have shown that, at least up to a loading of 1.1 $\mathrm{mmol} / \mathrm{g}$, the adsorption isotherm of $n$-pentane in ferrierite does not have any step or kink at $298 \mathrm{~K}^{12}$ Furthermore, we have measured an $n$-pentane loading of $1.0 \mathrm{mmol} / \mathrm{g}$ in ferrierite at $333 \mathrm{~K}$ at a pressure of at most $11 \mathrm{kPa}(20 \%$ of the saturation pressure of $n$-pentane at room temperature) using the procedure described in part 1 . Both observations imply that the occurrence of a step in the simulated adsorption isotherm of $n$-pentane does not agree with the experimental results. The possible origins of this discrepancy will be discussed later in this paper. First, we will present the other results obtained from the grandcanonical CB-MC simulations of the adsorption of propane up to $n$-hexane in ferrierite using the parameter set of Smit et al. and the Propane parameter set.

Location of Adsorbed Molecules in the Ferrierite Pore Structure. By determining the vector between the head and the tail of an alkane molecule, the location of the adsorbed molecules in the ferrierite pores can be analyzed. In this analysis, we have distinguished two adsorption sites: the ferrierite cage with its 8-ring windows and the 10-ring channels. The orientation of the vector indicates whether the molecule is adsorbed in the channels (z-direction) or in the cages ( $y$ direction). The fraction of molecules located in the ferrierite 8 -ring cages of the total amount of adsorbed molecules is given in Figure 5 as a function of the loading.

This figure shows that the $n$-hexane molecules are adsorbed exclusively in the 10-ring channels. In sharp contrast to $n$-hexane, propane has a strong preference for adsorption in the ferrierite 8-ring cages. The fraction of propane molecules located in the 8-ring cages decreases with increasing loadings, reaching finally a distribution over the channels and the cages of about 1 to 1 . Similarly to the propane molecules, the $n$-butane molecules are, even at low loadings, distributed over the channels and the cages. The $n$-butane molecules prefer, however, the channels. Initially, the adsorption of $n$-pentane takes place only in the channels. Adsorption in the ferrierite 8-ring cages does not occur before a loading of about 0.7 $\mathrm{mmol} / \mathrm{g}$ is reached. The $n$-butane and $n$-pentane distribution over the channel and cage becomes about 1 to 1 also at almost complete pore filling. It should be noted that the displayed 


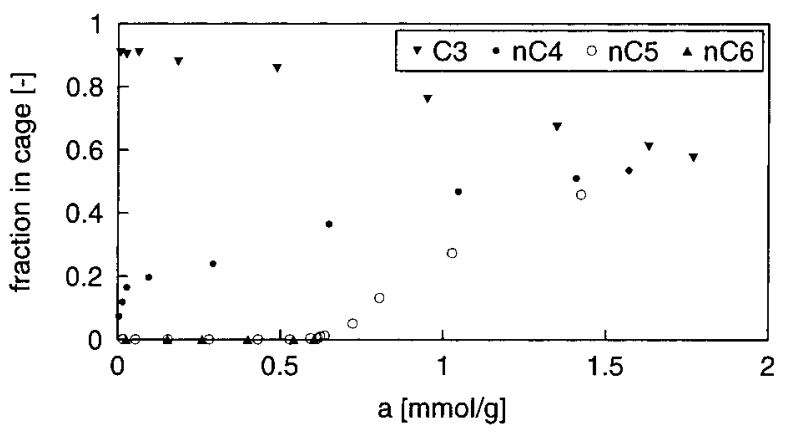

Figure 5. Fraction of alkane molecules located in the ferrierite 8-ring cage as a function of loading at $333 \mathrm{~K}$. Results are calculated using the Smit et al. parameter set.

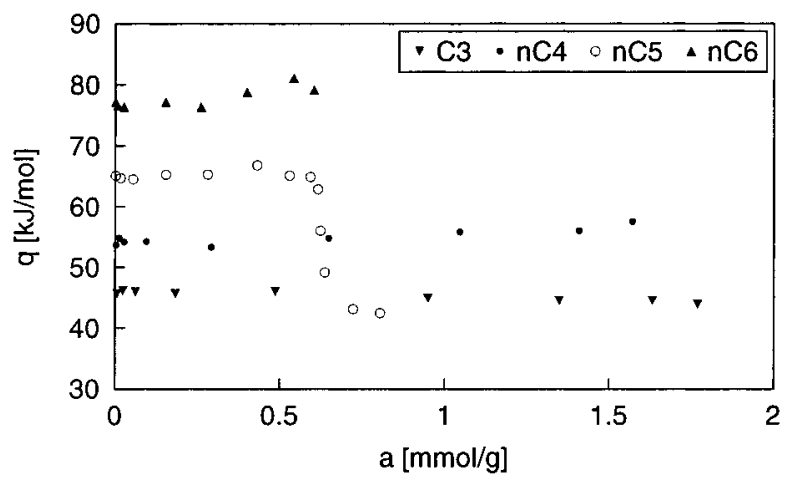

Figure 6. Isosteric heats of adsorption of alkanes as a function of loading at $333 \mathrm{~K}$. The heats of adsorption are calculated using the Smit et al. parameter set.

TABLE 2: Initial Isosteric Heats of Adsorption of Alkanes at $333 \mathrm{~K}$. Experimental Values Are Determined on H-FER; ${ }^{21}$ Calculated Values Are Determined on $\mathrm{SiO}_{2}$ FER

\begin{tabular}{lcc}
\hline & experimental $q_{\mathrm{st}}[\mathrm{kJ} / \mathrm{mol}]$ & calculated $q_{\mathrm{st}}[\mathrm{kJ} / \mathrm{mol}]$ \\
\hline propane & 52 & 46 \\
$n$-butane & 62 & 54 \\
$n$-pentane & 72 & 65 \\
$n$-hexane & 82 & 77
\end{tabular}

$n$-pentane loadings of 1.0 and $1.4 \mathrm{mmol} / \mathrm{g}$ were reached at pressures above the critical point. The results presented here on the location of the adsorbed molecules for propane, $n$ pentane, and $n$-hexane are in perfect agreement with the ${ }^{13} \mathrm{C}$ NMR results reported in part 1.12 Both simulations and NMR measurements show that $n$-butane at all loadings is distributed over the channels and the cages. While no preference is found by the NMR measurements, the simulations predict that $n$-butane adsorption in the 10-ring channels is preferred.

Energetics of Linear Alkanes Adsorbed in Ferrierite. The initial isosteric heats of adsorption calculated are given in Table 2 together with the experimental results of Eder et al. ${ }^{21}$ The heats of adsorption calculated are about 6-8 kJ/mol lower than the heats of adsorption measured experimentally on an acidic ferrierite sample. This is a reasonable difference since in the simulations we account only for the dispersive interactions between the zeolite framework and the alkane molecules and not for the specific interaction between the acid sites and the alkane molecules. It has been shown that this specific interaction contributes about $10 \mathrm{~kJ} / \mathrm{mol}$ for $\mathrm{H}-\mathrm{ZSM}-5$ and $6 \mathrm{~kJ} / \mathrm{mol}$ for H-FAU to the heat of adsorption. ${ }^{22}$

The change of the isosteric heat of adsorption with loading is displayed in Figure 6. The most remarkable feature of this figure is the drop in the heat of adsorption of $n$-pentane when loadings higher than $0.6 \mathrm{mmol} / \mathrm{g}$ are reached. This limit corresponds to the step in the adsorption isotherm and to the
TABLE 3: Number of Oxygen Atoms per Molecule That Are within a Repulsive Distance of the Alkane Molecule and the Corresponding Repulsive Energy per Molecule

\begin{tabular}{lcc}
\hline & $\begin{array}{c}\text { number of oxygen } \\
\text { atoms per molecule, } r<\sigma\end{array}$ & $U_{\text {repulsion }}[\mathrm{kJ} / \mathrm{mol}]$ \\
\hline propane in channels & 4.1 & 4.6 \\
propane in cages & 2.9 & 2.6 \\
$n$-butane in channels & 5.0 & 5.1 \\
$n$-butane in cages & 6.1 & 6.4 \\
$n$-pentane in channels & 6.9 & 7.0 \\
$n$-pentane in cages & 14.1 & 24.5
\end{tabular}

point where $n$-pentane starts to adsorb in the ferrierite 8-ring cages. This decrease in the heat of adsorption of $n$-pentane is also found experimentally. ${ }^{12}$ For both $n$-butane and $n$-hexane, an increase in the heat of adsorption with increasing loading can be observed which is the result of an increase of the interaction between the adsorbed molecules. This increase in the heat of adsorption amounts to about $5 \mathrm{~kJ} / \mathrm{mol}$. The interaction between the adsorbed molecules increases also for $n$-pentane and for propane with increasing loading. This results for $n$-pentane in an increase of the heat of adsorption up to a loading of $0.6 \mathrm{mmol} / \mathrm{g}$. However, the heat of adsorption of propane decreases slightly over the whole loading range with increasing loading due to a decrease of the interaction between the adsorbed molecules and the zeolite framework. This means that the dispersive interaction between the propane molecules and the framework oxygen atoms is more favorable in the ferrierite 8-ring cage than in the 10-ring channel. In the case of $n$-butane, the zeolite-alkane interaction is almost independent of the distribution of the molecules adsorbed. The preference found for adsorption in the 10-ring channels is mainly the result of a less positive torsion energy of the $n$-butane molecules in the channels compared to the $n$-butane molecules in the ferrierite cages. The torsion energy of $n$-pentane located in the ferrierite 8 -ring cage is also somewhat more positive $(5 \mathrm{~kJ} / \mathrm{mol})$ than the torsion energy of the $n$-pentane molecules in the 10-ring channel. But, the decrease in the heat of adsorption of $n$-pentane is mainly caused by a difference in interaction with the zeolite framework of $n$-pentane molecules adsorbed in the 10-ring channel and $n$-pentane molecules adsorbed in the ferrierite 8-ring cage.

The three parameters discussed above, the intermolecular interaction, the zeolite-chain interaction, and the torsion energy of the alkane molecules, are mainly responsible for the change of the heats of adsorption with loading. The bond bending and intramolecular energies vary only slightly with the loading of $n$-alkane in the zeolite. The enthalpy of the bulk phase deviates only significantly from the ideal gas value for the simulations performed near the critical point of $n$-pentane.

We have analyzed the distances between the framework oxygen atoms and the alkane united atoms to elucidate the behavior of the interaction between the zeolite framework and the alkane molecules as a function of the location of the adsorbing molecules. Table 3 displays the number of oxygen atoms that give per molecule a repulsive interaction (these are the oxygen atoms that are within a distance smaller than the size parameter $\sigma$ used) together with the contribution of these repulsive interactions to the total interaction between the zeolite framework and the alkane molecules. The results are separated according to the molecule location, 8-ring cage or 10-ring channel, and are obtained from the configurations at the highest pressure simulated. Results from lower pressure simulations are comparable to these.

It is clear that the contribution of the repulsive interactions is much higher for the $n$-pentane molecules located in the ferrierite cages than for those in the channels. In contrast to 


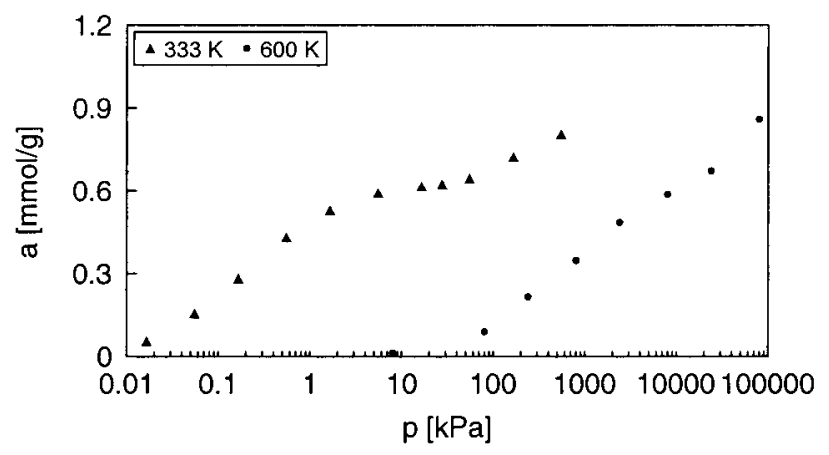

Figure 7. Adsorption isotherms of $n$-pentane at 333 and $600 \mathrm{~K}$. The isotherms are calculated using the Smit et al. parameter set.

$n$-pentane, the repulsive interactions are about equal for $n$-butane in the channels and $n$-butane in the cages. This shows that the decrease of the heat of adsorption when $n$-pentane adsorbs in the ferrierite cages is mainly caused by the larger repulsion in those cages. This repulsion is due to the limited volume of the ferrierite 8-ring cage so that an $n$-pentane molecule adsorbed in this cage has to be within an unfavorable distance of several oxygen atoms. As a result of its smaller chain length, the number of repulsive interactions is much smaller for $n$-butane, which corresponds to the absence of a decrease in the heat of adsorption. The much higher repulsive interactions of the $n$-pentane molecules than of the $n$-butane molecules in the ferrierite 8-ring cage is in agreement with our observation in the ${ }^{13} \mathrm{C}$ NMR experiments that the signal of the methyl carbon atoms of $n$-pentane occurs at much higher chemical shifts than the $n$-butane methyl carbon atoms in the ferrierite 8 -ring cage. ${ }^{12}$ In this context, it is noteworthy that the methyl carbon atoms of propane, $n$-butane, and $n$-pentane located in the ferrierite 8-ring cage have significantly more repulsive interactions than the methylene carbon atoms. The results given in Table 3 show also that, in contrast to $n$-butane and $n$-pentane, propane molecules located in the 10-ring channels suffer somewhat more from repulsive interactions than propane molecules in the 8-ring cages. This lower contribution of repulsive contributions is partly responsible for the more favorable interaction with the zeolite framework of the propane molecules in the ferrierite cages.

Adsorption Isotherm at $600 \mathrm{~K}$. The adsorption isotherm of $n$-pentane is simulated at $600 \mathrm{~K}$ to investigate the influence of temperature on the adsorption behavior of $n$-pentane. Simulations at high temperatures are furthermore interesting because of the catalytic processes that are carried out at these temperatures and because of the difficulty of performing adsorption experiments under these reaction conditions. The $n$-pentane isotherm at $600 \mathrm{~K}$ is displayed in Figure 7 together with the isotherm simulated at $333 \mathrm{~K}$. It is clear that the step in the simulated adsorption isotherm is much smaller at $600 \mathrm{~K}$ than at $333 \mathrm{~K}$. This is in agreement with the fact that this step is caused by a decrease of the heat of adsorption. This temperature dependence reflects nicely the difference in explanation for the special adsorption behavior of $n$-pentane in ferrierite and of $n$-hexane or $n$-heptane in ZSM-5. In contrast to the adsorption of $n$-pentane in ferrierite, the step in the adsorption isotherm of $n$-hexane or $n$-heptane in ZSM-5 is caused by differences in adsorption entropy. $5,23-25$ As a result of this entropic origin, the step in the $n$-hexane adsorption isotherm in ZSM-5 becomes more pronounced at higher temperatures. . $^{56}$

The higher adsorption temperature influences also slightly the location of the $n$-pentane molecules in the pores of ferrierite.
At a temperature of $333 \mathrm{~K}$, there are no $n$-pentane molecules found at all in the ferrierite 8-ring cage at loadings lower than $0.53 \mathrm{mmol} / \mathrm{g}$. However at $600 \mathrm{~K}$, we find the first $n$-pentane molecules in the ferrierite 8-ring cage already at a loading of $0.35 \mathrm{mmol} / \mathrm{g}$. There is also a pronounced decrease of the $n$-pentane heat of adsorption at $600 \mathrm{~K}$.

Comparison between Different Parameter Sets. As mentioned previously, the predicted step in the $n$-pentane adsorption isotherm is not in agreement with the experimental results. One possible reason is the use of a rigid zeolite lattice during the simulations. Although this is a very common assumption in theoretical studies on static adsorption properties, its validity becomes questionable when the adsorbate molecules fit tightly in the zeolite pores. Pronounced lattice distortions are, for example, known to occur upon adsorption of aromatic molecules in ZSM-5. It is clear, from the results presented in this work, that the $n$-pentane molecules fit tightly in the ferrierite 8-ring cage. However, for a number of reasons, we do not believe that the assumption of a rigid lattice is the main cause of the observed discrepancy. The structural parameters of ferrierite are, like other zeolites, dependent on the aluminum content of the framework. It was reported by Wise that the $a_{0}$ cell parameter, corresponding to the smallest diameter of the 8-ring window of the ferrierite cage, is proportional to the number of aluminum atoms per unit cell. ${ }^{27}$ The $a_{0}$ cell parameter and the unit cell volume of all-silica ferrierite are about $4 \%$ smaller than the corresponding values determined by Vaughan on a ferrierite sample with 5.5 aluminum atoms per unit cell. ${ }^{28,29}$ These two parameters of the all-silica ferrierite are only less than $1 \%$ larger when the template molecules, pyridine and 1-amino- $n$-propane, are still present in the framework. ${ }^{29}$ This suggests that the cell parameters are more dependent on the aluminum content than on the presence of template molecules in the pores. In this context, it should further be remembered that the all-silica structure used in the simulations is based on structural data determined on a ferrierite sample that contains more aluminum than the samples used in the experiments. It is therefore more likely that, on the basis of the zeolite structure, we underestimate repulsion effects of $n$-pentane in the ferrierite 8-ring cage rather than that we overestimate them.

Besides the use of a rigid lattice, we believe that the imperfection of the parameters used to describe the interaction between the framework oxygen atoms and the $n$-alkane pseudoatoms is an important reason for the observed discrepancy. These parameters are derived from adsorption data of linear alkanes in silicalite and might therefore be less correct to predict the adsorption in ferrierite. To illustrate our point of view, we have performed some calculations with the size and energy parameters that have been derived by June et al. ${ }^{13}$ The most important difference with the parameters of Smit et al. is the slightly, about $8 \%$, smaller size parameter $\sigma$. The adsorption isotherms of $n$-butane and $n$-pentane in ferrierite at $333 \mathrm{~K}$ calculated with the parameters of June et al. are compared to the experimental isotherms in Figure 8 . It is evident that the $n$-butane and especially the $n$-pentane loadings are, at a given pressure, now overestimated by the calculations. In contrast to the experimental results, the $n$-butane and $n$-pentane isotherms calculated do not cross each other. This is due to a greater overestimation of the $n$-pentane loading than of the $n$-butane loading. The results in Figure 8 show also that a small change in the size parameter is enough to make the huge step in the calculated $n$-pentane isotherm disappear. In other words, the occurrence (and width) of the step in the calculated $n$-pentane isotherm is very sensitive to the size parameter used. Also the $n$-hexane 


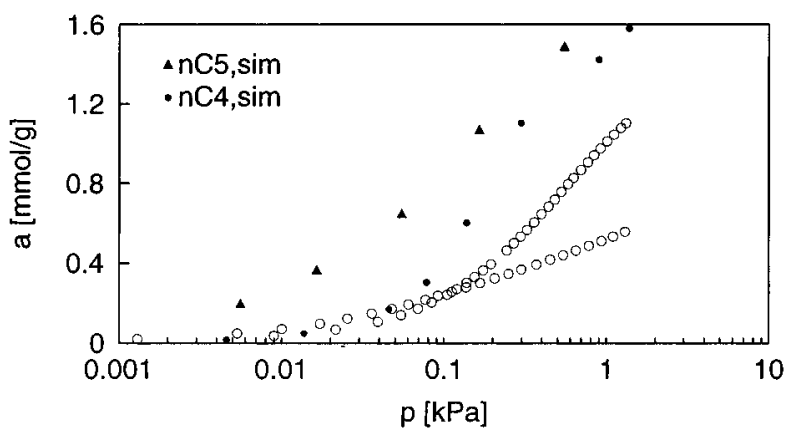

Figure 8. Simulated and experimental adsorption isotherms of $n$-butane and $n$-pentane in FER at $333 \mathrm{~K}$. The simulated isotherms are determined on $\mathrm{SiO}_{2}$ FER using the June et al. parameter set; the experimental isotherms are determined on H-FER. ${ }^{21}$

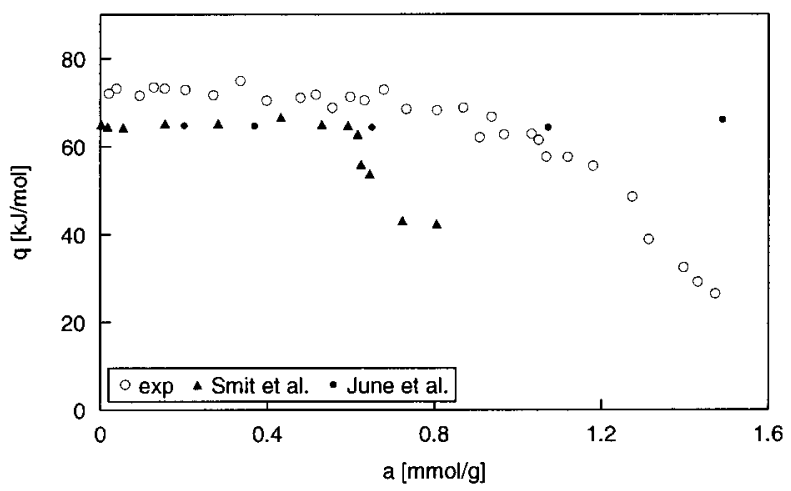

Figure 9. Simulated isosteric heat of adsorption of $n$-pentane using the Smit et al. and using the June et al. parameter set in $\mathrm{SiO}_{2}$ FER at $333 \mathrm{~K}$ and the experimental isosteric heat of adsorption of $n$-pentane in H-FER at $298 \mathrm{~K}^{12}$

loading is, at a given pressure, overestimated when the June et al. parameters are used. The calculations even predict that, at pressures of $0.019 \mathrm{kPa}$ and higher, $n$-hexane adsorbs also in the ferrierite 8-ring cages. When the Smit et al. parameters are used, we do not even find any $n$-hexane molecules in the ferrierite 8-ring cage at a pressure as high as $6300 \mathrm{kPa}$. The described overestimation of the loading, when the June et al. parameters are used in the calculations, was also found for the $n$-hexane isotherm in silicalite. The $n$-butane isotherm in silicalite was however predicted correctly with the parameters of June et al. ${ }^{14}$

The $n$-pentane heat of adsorption calculated as a function of loading is also influenced by the choice of parameters. The results calculated with the Smit et al. and with the June et al. parameters (at $333 \mathrm{~K}$ ) are compared to the experimentally measured heat of adsorption (at $298 \mathrm{~K})^{12}$ in Figure 9. In contrast to the experimental results and to the results calculated with the Smit et al. parameters, the heat of adsorption as calculated with the parameters of June et al. does not show a decrease when higher loadings are reached. This shows that, when the latter parameters are used, the $n$-pentane molecules in the ferrierite 8-ring cage do not have many oxygen atoms within a repulsive distance. The latter is quite understandable when we take into account that the interaction calculated between a framework oxygen atom and an $n$-alkane united atom becomes repulsive when the distance between them is smaller than the size parameter $\sigma$. While the difference in repulsive interaction between an $n$-pentane molecule in the cage and an $n$-pentane molecule in the channel was $18 \mathrm{~kJ} / \mathrm{mol}$ when the Smit et al. parameters are used, it is only $4 \mathrm{~kJ} / \mathrm{mol}(6 \mathrm{~kJ} / \mathrm{mol}$ for the molecules in the cages; $2 \mathrm{~kJ} / \mathrm{mol}$ for the molecules in the channels) with the June et al. parameters. Both the shape of the $n$-pentane adsorption isotherm and heat of adsorption as a function of loading calculated are very sensitive to the chosen size parameter in the simulations due to the narrow fit of the $n$-pentane molecules in the ferrierite 8 -ring cage. The adsorption of $n$-pentane in ferrierite can therefore be used to set the limits of the size parameter describing the interaction between the zeolite framework and the alkane molecules.

The location of the $n$-butane and $n$-pentane molecules in the ferrierite pores is also markedly influenced by the choice of the parameters. We now find with the June et al. parameters at all loadings some $n$-pentane molecules in the ferrierite 8-ring cage (for example $1 \%$ of the molecules at $0.25 \mathrm{mmol} / \mathrm{g}$ ), while the percentage of $n$-butane molecules adsorbed in the ferrierite 8 -ring cage calculated is $43 \%$ at zero coverage.

\section{Conclusions}

The results obtained from configurational-bias Monte Carlo calculations in the grand-canonical ensemble on the adsorption of linear alkanes are generally in good agreement with experimental results regarding adsorption isotherms, isosteric heat of adsorption, and the location of the molecules in the ferrierite pore structure. The simulation results have provided a better physical understanding of the chain length effects in the adsorption properties of linear alkanes in ferrierite. Finally, through comparison of the simulation results with the experimental results some of the parameters used in the calculations have been tested.

Adsorption of propane is more favorable in the 8-ring cage than in the 10-ring channel due to a combination of lower repulsive and higher attractive interactions with the zeolite framework. Propane adsorbs therefore preferentially in this cage resulting initially in a distribution of the adsorbed propane molecules over the cages and the channels of about 9:1. The isosteric heat of adsorption of propane decreases with increasing loading due to increased adsorption in the less favorable 10ring channels. The heat of adsorption of $n$-butane increases slightly with increasing loading due to an increased interaction between the $n$-butane molecules. Adsorption in the 10-ring channel is preferred as a result of the slightly lower repulsive interactions and the less positive torsion energy of the $n$-butane molecules in the 10-ring channels compared to the molecules in the ferrierite 8-ring cage.

The adsorption of $n$-pentane in the ferrierite 8 -ring cage is hindered due to the limited volume of this cage and results in a large number of repulsive interactions between the zeolite framework and the $n$-pentane molecules. As a result, there is a pronounced decrease in the heat of adsorption when $n$-pentane adsorbs in the ferrierite 8-ring cage. The lower heat of adsorption of $n$-pentane in the ferrierite 8 -ring cage is in agreement with the $n$-pentane heat of adsorption as a function of loading measured experimentally combined with the locations obtained from the ${ }^{13} \mathrm{C}$ NMR measurements. ${ }^{12}$

The repulsive interactions of $n$-pentane in the ferrierite 8-ring cage calculated, and consequently the heat of adsorption, is very dependent on the size parameter used in the Lennard-Jones potential describing the interaction between the zeolite framework oxygen atoms and the $n$-alkane pseudoatoms. When a size parameter of $3.64 \AA$ is used, a decrease in heat of adsorption upon adsorption of $n$-pentane in the 8 -ring cage is predicted. The lower heat of adsorption of $n$-pentane in the 8-ring cage compared to the 10-ring channel is in agreement with the experimental results. This decrease in heat of adsorption of $n$-pentane is absent when a size parameter of $3.364 \AA$ is used. The latter is the result of an underestimation of the repulsive 
interactions between the $n$-pentane molecules in the ferrierite cage and the zeolite framework due to the smaller size parameter used. The shape of the $n$-pentane adsorption isotherm calculated is also determined by the choice of the size parameter. While the use of the smallest size parameter of $3.364 \AA$ results in a smooth isotherm, a huge step in the isotherm is predicted when a size parameter of $3.64 \AA$ is used. Since a step is absent in the isotherm measured experimentally, we can conclude that a size parameter between 3.364 and $3.64 \AA$ is needed for a correct description of the adsorption properties of linear alkanes in ferrierite.

Acknowledgment. W.J.M.v.W. is indebted to the Stichting Scheikundig Onderzoek in Nederland (SON). Netherlands Institute for Research in Catalysis (NIOK) publication \#TUE 98-5-04.

\section{References and Notes}

(1) Part of this work was communicated previously: van Well, W. J. M.; Cottin, X.; de Haan, J. W.; van Santen, R. A.; Smit, B. Angew. Chem., Int. Ed. Engl. 1988, 37, 1081.

(2) Smit, B.; Siepmann, J. I. Science 1994, 264, 1118.

(3) Smit, B.; Siepmann, J. I. J. Phys. Chem. 1994, 98, 8442.

(4) Smit, B. J. Phys. Chem. 1995, 99, 5597.

(5) Smit, B.; Maesen, T. L. M. Nature 1995, 374, 42

(6) Maginn, E. J.; Bell, A. T.; Theodorou, D. N. J. Phys. Chem. 1995, 99, 2057.

(7) Bates, S. P.; van Well, W. J. M.; van Santen, R. A.; Smit, B. J. Am. Chem. Soc. 1996, 118, 6753.

(8) Bates, S. P.; van Well, W. J. M.; van Santen, R. A.; Smit, B. J. Phys. Chem. 1996, 100, 17573.
(9) Bandyopadhyay, S.; Yashonath, S. J. Chem. Phys. 1996, 105, 7223. 5675 .

(10) Bandyopadhyay, S.; Yashonath, S. J. Phys. Chem. B 1997, 101

(11) Mooiweer, H. H.; de Jong, K. P.; Kraushaar-Czarnetzki, B.; Stork, W. H. J.; Krutzen, B. C. H. Stud. Surf. Sci. Catal. 1994, 84, 2327.

(12) van Well, W. J. M.; Cottin, X.; de Haan, J. W.; van Hooff, J. H. C.; van Santen, R. A.; Smit, B.; Nivarthy, G.; Lercher, J. A. J. Phys. Chem. $B$ 1998, 102, 3945.

(13) June, R. L.; Bell, A. T.; Theodorou, D. N. J. Phys. Chem. 1992, $96,1051$.

(14) Smit, B. Mol. Phys. 1995, 85, 153.

(15) Frenkel, D.; Smit, B. Understanding Molecular Simulations: From Algorithms to Applications; Academic Press: Boston, 1996.

(16) Siepmann, J. I.; Karaborni, S.; Smit, B. Nature 1993, 365.

(17) Vaughan, P. A. Acta Crystallogr. 1966, 21, 983.

(18) Wood, G. B.; Panagiotopoulos, A. Z.; Rowlinson, J. S. Mol. Phys. 1988, 63, 49 .

(19) Vuong, T.; Monson, P. A. Langmuir 1996, 12, 5425.

(20) Lee, B. I.; Kesler, M. G. AIChE Journal 1975, 21, 510.

(21) Eder, F.; Lercher, J. A. J. Phys. Chem. B 1997, 101, 1273.

(22) Eder, F.; Lercher, J. A. Zeolites 1997, 18, 75.

(23) van Well, W. J. M.; Wolthuizen, J. P.; Smit, B.; van Hooff, J. H. C.; van Santen, R. A. Angew. Chem., Int. Ed. Engl. 1995, 34, 2543.

(24) van Well, W. J. M.; Wolthuizen, J. P.; Smit, B.; van Hooff, J. H. C.; van Santen, R. A. Stud. Surf. Sci. Catal. 1997, 105, 2347.

(25) Olson, D. H.; Reischman, P. T. Zeolites 1996, 17, 434.

(26) Richards, R. E.; Rees, L. V. C. Langmuir 1987, 3, 335.

(27) Wise, W. S. Am. Mineral. 1976, 61, 60.

(28) Morris, R. E.; Weigel, S. J.; Henson, N. J.; Bull, L. M.; Janicke, M. T.; Chmelka, B. F.; Cheetham, A. K. J. Am. Chem. Soc. 1994, 116 11849.

(29) Lewis, J. E.; Freyhard, C. C.; Davis, M. E. J. Phys. Chem. 1996, $100,5039$. 